\title{
MEG Decoding Across Subjects
}

\author{
Emanuele Olivetti, Seyed Mostafa Kia and Paolo Avesani \\ NeuroInformatics Laboratory (NILab), \\ Bruno Kessler Foundation, Trento, Italy \\ Centro Interdipartimentale Mente e Cervello (CIMeC), \\ University of Trento, Italy
}

September 18, 2018

\begin{abstract}
Brain decoding is a data analysis paradigm for neuroimaging experiments that is based on predicting the stimulus presented to the subject from the concurrent brain activity. In order to make inference at the group level, a straightforward but sometimes unsuccessful approach is to train a classifier on the trials of a group of subjects and then to test it on unseen trials from new subjects. The extreme difficulty is related to the structural and functional variability across the subjects. We call this approach decoding across subjects. In this work, we address the problem of decoding across subjects for magnetoencephalographic (MEG) experiments and we provide the following contributions: first, we formally describe the problem and show that it belongs to a machine learning sub-field called transductive transfer learning (TTL). Second, we propose to use a simple TTL technique that accounts for the differences between train data and test data. Third, we propose the use of ensemble learning, and specifically of stacked generalization, to address the variability across subjects within train data, with the aim of producing more stable classifiers. On a face vs. scramble task MEG dataset of 16 subjects, we compare the standard approach of not modelling the differences across subjects, to the proposed one of combining TTL and ensemble learning. We show that the proposed approach is consistently more accurate than the standard one.
\end{abstract}

\section{Introduction}

Predicting the mental state of a subject from concurrent neuroimaging data is a data analysis approach usually called brain decoding. The subject is presented a stimulus, e.g. a visual cue, and the related brain activity is recorded from multiple sensors. The recorded data, together with the category of the stimulus, are denoted as trial. During an experiment multiple trials are collected and in the brain decoding analysis phase, part of the data, i.e. the train set, 
is used to build a classifier, i.e. a function that predicts the stimulus from the brain activity. The remaining part of the data are used to estimate how accurate the classifier is, i.e. to test its generalisation ability [1]. Accurate classification is considered evidence that stimulus-related information is present in the data, a conclusion that shed light on the relation between the mental process of interest and its neural correlates. In the following, we refer to the case magnetoencephalographic (MEG) data and defer the general case of functional neuroimaging data to future work.

Brain decoding is usually performed at the level of each single subject and inference at the group-level is frequently conceived as the analysis of the set of the single subject results, e.g. a one-sample $t$-test over the set of single-subject accuracies (See 2 for a critical discussion). Ideally, training a classifier on the trials from a set of subjects and testing the classifier on trials from other unseen subjects is a meaningful process to make inference at the group level. Unfortunately this approach is technically difficult because of the structural and functional differences of the brain across subjects together with the inherent variability of the MEG measurements due, for example, to changes in environmental variables. The effect of this variability is that the underlying probability distribution of the trials changes from subject to subject violating the assumption of a single underlying generative process 11 between the train set and the test set, which is the cornerstone of statistical learning and, in general, of inference. In practical terms, it is common experience to observe that the average accuracy of the classifiers trained and tested on each individual subject is much greater than that of a classifier trained on pooling trials of a set of subjects and tested on trials from other subjects. We provide empirical evidence of this fact in Section 3 ,

To the best of our knowledge, the literature proposing solutions to the problem of MEG decoding across subjects for inferential purpose is at very early stage. Most of the effort is in creating a representation of the MEG data, i.e. a new feature space, that is as homogeneous as possible across subjects. A recent example is in 3 .

Other fields of research are related, to some extent, to the problem of MEG decoding across subjects. They can be divided into three main different areas:

- Machine Learning literature dealing with training and testing on different feature spaces and/or different distributions - a general paradigm known as transfer learning. See 4 for a recent review. To the best of out knowledge, in this community no applications have been presented in the context of MEG data analysis.

- Brain-computer interface (BCI) literature on between-subject learning from EEG data (see for example [5]). In this setting, data from other subjects is used to improve the calibration of BCI devices when used on a new subject. The stimulus information is required for at least some

\footnotetext{
${ }^{1}$ The specific assumption is that, for each category of stimulus, the concurrent neuroimaging data are independently and identically distributed (i.i.d.).
} 
trials of the new subject. This requirement is different from our setting where no stimulus information is available on test subjects. Notice that this technical difference leads to a completely different approach during data analysis.

- Literature about decoding across subjects from functional magnetic resonance imaging (fMRI) data. To the best of our knowledge, the two leading directions are hyperalignement (see for example [6]) and multi-task learning (see for example [7]). Both differ from our setting: hyperalignement requires an additional recording from all subjects with identical rich stimulation. In multi-task learning it is assumed that some stimulus information is available on test data 2 .

In this work, we propose a formal definition of the problem of decoding across subjects, and we claim that it is an instance of transfer learning, and more precisely of transductive transfer learning (TTL). Transfer learning aims at transferring knowledge from the train set to the test set, assuming they differ in some aspects. Transductive transfer learning is focused on problems where class-labels are not available for the test set, but the unlabelled set of recordings of the test set can be used to transfer knowledge acquired from the (classlabelled) train set. As a further contribution, we present a simple and practical TTL solution based on the covariate shift assumption. A final contribution is the use of the ensemble learning principle to enhance the training process of the classifier on the different datasets of the training subjects. We propose to use an ensemble learning technique called stacked generalization and motivate why ensemble learning is useful for our setting. The combination of TTL and ensemble learning is the method that we propose for decoding across subject. We show experimental evidence of the efficacy of stacked generalization and simple covariate shift on a face vs. scramble multisubject MEG dataset.

The article is structured as follows: in Section 2, we formally describe the problem of decoding across subjects and illustrate the building blocks of the proposed approach: transfer learning, transductive transfer learning, covariate shift and stacked generalization. In Section 3, we show experimental evidence of the efficacy of the proposed method on real MEG data and multiple subjects. In Section 4, we discuss our results and draw conclusions.

\section{Methods}

In this section, we formally describe decoding across subjects as a transfer learning problem and more specifically as a transductive transfer learning problem. Then we introduce the necessary building blocks of the proposed solution, i.e. a simple TTL algorithm based on the covariate shift assumption and the stacked generalization technique of ensemble learning.

\footnotetext{
2 [7] extends this approach so that this assumption is not strictly necessary.
} 
In the following, we assume that MEG data are already preprocessed. We briefly summarise our standard pre-processing steps in Section 3 when discussing experiments.

\subsection{Transfer Learning}

Here we introduce the basic concepts of transfer learning with focus on our application of decoding across subjects. Let $X \in \mathcal{X}$ be the MEG recording of a single trial $(X, y)$, where $y \in \mathcal{Y}$ represents the stimulus category presented to the subject. In our case $\mathcal{X}=\mathbb{R}^{d C}$, where $C$ is the number of channels of the MEG system and $d$ is the number of timesteps recorded. Moreover, we assume a binary stimulus, i.e. $\mathcal{Y}=\{0,1\}$. Let $P(X)$ be the marginal probability distribution of $X$. Following [4] we call $\mathcal{D}=\{\mathcal{X}, P(X)\}$ a domain, which in our case is a given subject from which we record MEG data. Given a domain, let $f: \mathcal{X} \mapsto \mathcal{Y}$ be the predictive target function that can be approximated from observed data. Then, for a given domain $\mathcal{D}$, a task is defined as a predictive function and its output space, i.e. $\mathcal{T}=\{\mathcal{Y}, f\}$. In our case, a task could be discriminating trials when the visual stimuli are face vs. scrambled face, or another task could be or face vs. house.

We assume to have trials recorded from a source domain $S$ and from a target domain $T$. In our application, this means that we have trials recorded from at least two different subjects. Moreover, in order to provide a general definition of transfer learning, we assume that we have a source task $\mathcal{T}_{S}$ and a target task $\mathcal{T}_{T}$. Following the example above, these two tasks could be face vs. scrambled face and face vs. house. Then, as stated in 4, "transfer learning aims to help improve the learning of the target predictive function $f_{T}$ in $\mathcal{D}_{T}$ using the knowledge in $\mathcal{D}_{S}$ and $\mathcal{T}_{S}$, where, in general, $\mathcal{D}_{S} \neq \mathcal{D}_{T}$ and $\mathcal{T}_{S} \neq \mathcal{T}_{T}$ ".

Transfer learning is a general area of research that contains different settings, each related to a specific sub-field of machine learning. Traditional machine learning is the case in which $\mathcal{D}_{S}=\mathcal{D}_{T}$ and $\mathcal{T}_{S}=\mathcal{T}_{T}$. In [4] a taxonomy of all the settings is illustrated and it is shown that main differences between them depend on: how much domains and tasks differ from source to target, and whetheror not class labels are available in the source domain or in the target

domain. The specific case of interest for this work is defined as follows: we have MEG data from two different subjects on the same decoding task. We use the first to train a classifier and the second to test it, i.e. class-labels are not available on the second subject. In other words, our problem has two different but related domains, i.e. the two subjects, and the tasks is the same in both cases, e.g. decoding face vs. scrambled face. Moreover class-labels are available in the source domain but not in the target domain. This specific setting of the transfer learning problem is called transductive transfer learning.

\subsection{Transductive Transfer Learning (TTL)}

According to 4, transductive transfer learning (TTL) aims to improve the learning of $f_{T}$ in $\mathcal{D}_{T}$ when $\mathcal{D}_{S} \neq \mathcal{D}_{T}$ and $\mathcal{T}_{S}=\mathcal{T}_{T}$ and when unlabeled data 
from the target domain are available at training time. The term transductive stresses both the identical task and the availability of unlabelled data.

The TTL setting can be divided into two categories:

1. $\mathcal{X}_{S} \neq \mathcal{X}_{T}$, i.e. source and target have different features spaces.

2. $P\left(X_{s}\right) \neq P\left(X_{T}\right)$, i.e. source and target share the same feature space, but the respective marginal probability distributions differ.

Most TTL approaches available in the literature belong to the second category. They go under the names of covariate shift (see for example [8]), domain adaptation (see for example [9]) or sample selection bias (see for example [10]). To the best of our knowledge, transferring knowledge between different representations, i.e. the first category, has no general solutions but only a few domain-specific ones in domains different from ours. See 4 for a brief review.

In this work, we assume that $\mathcal{X}_{S}=\mathcal{X}_{T}$ and that the difference between the data recorded from the source and target subjects during the same task is expressed just by $P\left(X_{s}\right) \neq P\left(X_{T}\right)$. This assumption is both meaningful and convenient. It is meaningful because, as long as the training subject is similar to the test subject, direct learning transfer can happen. It is also convenient because there are some simple solutions already available in the literature. In the following we describe the simplest one, described by multiple authors, which is based on the idea of importance sampling. See for example [10, 8].

\subsubsection{Simple Covariate Shift}

In the empirical risk minimization framework, learning is the process of minimizing the loss function over train data:

$$
f^{*}=\underset{f \in \mathcal{F}}{\operatorname{argmin}} \frac{1}{n} \sum_{i=1}^{n} l\left(f, x_{i}, y_{i}\right)
$$

where $l()$ is a loss function when learning a function $f \in \mathcal{F}$ from a dataset $\left\{\left(x_{1}, y_{1}\right), \ldots,\left(x_{n}, y_{n}\right)\right\}$. If the train dataset is drawn from $P_{S}(X, Y)$ but we are interested in predictions when the test data come from $P_{T}(X, Y)$, then each term can be penalized according to how likely each trial belongs to the target domain:

$$
f^{*}=\underset{f \in \mathcal{F}}{\operatorname{argmin}} \frac{1}{n} \sum_{i=1}^{n} \frac{P_{T}\left(x_{i}, y_{i}\right)}{P_{S}\left(x_{i}, y_{i}\right)} l\left(f, x_{i}, y_{i}\right) .
$$

The covariate shift assumption is that $P_{S}(Y \mid X)=P_{T}(Y \mid X)$, then $\frac{P_{T}\left(x_{i}, y_{i}\right)}{P_{S}\left(x_{i}, y_{i}\right)}=$ $\frac{P_{T}\left(x_{i}\right)}{P_{S}\left(x_{i}\right)}$. Then, the risk minimization problem becomes:

$$
f^{*}=\underset{f \in \mathcal{F}}{\operatorname{argmin}} \frac{1}{n} \sum_{i=1}^{n} \frac{P_{T}\left(x_{i}\right)}{P_{S}\left(x_{i}\right)} l\left(f, x_{i}, y_{i}\right) .
$$

Among the various ways to estimate $\frac{P_{T}\left(x_{i}\right)}{P_{S}\left(x_{i}\right)}$, a simple one is to set up a new classification problem to discriminate trials belonging to the source domain from those of the target domain, which requires just unlabeled data. 


\subsection{Ensemble Learning: Stacked Generalization}

It is common experience that, when single-subject decoding is accurate, decoding across subject, by just pooling all trials of train subjects together, is not accurate on trials from test subjects. As previously discussed, the cause of this issue is the variability across subjects. This situation shares analogy with ensemble learning, where multiple classifiers are trained from portions of the train set and then combined to create a single prediction on test data. The underlying ideas of ensemble learning are mainly two: first, each classifier in the ensemble tries to capture specific aspects of the train set. Second, the additional step of combining the classifiers of the ensemble tries to reduce the instability of the prediction of each of them. In short, diversity of classifiers and stability of the combined predictions are the two key elements of ensemble learning. In our problem of decoding across subjects, the set of trials of the train subjects is naturally partitioned in a way that training one classifier for each subject is expected to well represent the diversity within the train data. For this reason, we claim that combining these classifiers can be more effective than ignoring the differences and pooling all subjects.

In the vast literature of ensemble learning, multiple approaches have been proposed, such as bagging, boosting, Bayesian model averaging and stacked generalization. A reference book for this topic is [11. Among the many techniques available in this field of research, here we propose to use stacked generalization, also known as stacking classifiers, which aims at learning the combination of classifiers. See 12, 13] for a detailed introduction to stacked generalization. Here, we briefly introduce the basic procedure in the context of the problem of decoding across subjects.

The procedure of stacked generalization is divided in the following steps:

1. Train a set of classifiers on (portions of) the train data. These classifiers are called first-level classifiers.

2. Collect the output of each classifier on each trial of the train and of the test data. These outputs are called first level predictions.

3. Create a new second-level dataset with the vector of first-level predictions for each trial. Care has to be taken so that the predicted value of a given trial comes from classifiers which were not trained on that trial, e.g. through cross-validation.

4. The class-labels of the second-level dataset are the same as the initial dataset.

5. A second-level classifier is trained on the portion of the second-level dataset related to the train subjects in order to learn how to combine the first-level predictions.

6. The second level classifier is used to predict the class-labels of the test data as represented in the second-level dataset. 
In this work, we claim that creating each first-level classifier on the data of one subject only, is an effective way to ensure diversity which is a key element for the success of ensemble learning. Moreover, this approach implicitly assumes that the test set can be represented as a combination of the different patterns observed in the data of the training subjects, which is a desirable property for our specific problem.

As a final step, we propose to combine covariate shift and stacked generalization in the following way: once the second level classifier is created, each instance related to the training subjects is weighted according to the simple re-weighting procedure described in 2.2.1.

\section{Experiments}

We tested the proposed method on an MEG dataset where subjects were presented visual stimuli about famous faces, unfamiliar faces and scrambled faces. The dataset is from a multi-modal study described in [14] and consists of 16 subjects. We refer the reader to the original study for a comprehensive description of the dataset. We created a balanced face vs. scramble dataset by drawing at random from the trials of famous and unfamiliar faces in equal number to that scrambled faces. The dataset of each subject consisted of $\approx 580$ trials, so the entire dataset consisted of 9414 trials. We high-pass filtered the raw data at $1 \mathrm{~Hz}$, downsampled to $200 \mathrm{~Hz}$, and epoched each trial to the first $500 \mathrm{~ms}$ after stimulus onset. Then each trial consisted of 100 timepoints for each of the 306 MEG channels, which we concatenated into a vector of $100 \times 306=30600$ elements that was the input of the classification algorithms.

First, we attempted single-subject decoding, using logistic regression with $\ell_{1}$ penalization. The column "single" in Table 1 shows the 6 -fold cross-validated $(\mathrm{CV})$ accuracies. The results show that high accuracy could be reached in all cases, with an average accuracy of 0.82 and a range of $0.70-0.90$.

We conducted a leave-one-subject out cross-validation with the three different methods described in Section 2. The results are reported in Table11and the corresponding columns are indicated in the following. The first method (column "pool") consisted in just pooling all trials of all subjects and using logistic regression with $\ell_{1}$ penalization. We observe that the average accuracy drops to 0.62 , which is a 0.2 reduction with respect to the single-subject decoding accuracy.

The second method (column "SG" in Table 1) is based on stacked generalization where both the first-level and second-level classifiers were again logistic regression. The average accuracy is 0.65 and consistently greater that pooling over almost all subjects. The third method (column "SG+CS" in Table1) is the same as the second method but additionally each trial of the second-level dataset was weighted with a coefficient computed according to the simple covariate-shift technique described in Section 2.2.1. The classifier used to compute the weight of each trial was again logistic regression. The average accuracy is 0.67 , and is consistently greater or equal to that of plain stacked generalization over almost 


\begin{tabular}{c|c||c|c|c|} 
Subj. & single & pool & SG & SG+CS \\
\hline 1 & 0.82 & 0.62 & 0.67 & $\mathbf{0 . 7 1}$ \\
2 & 0.75 & 0.64 & 0.63 & $\mathbf{0 . 6 5}$ \\
3 & 0.82 & 0.60 & 0.59 & $\mathbf{0 . 6 1}$ \\
4 & 0.86 & 0.70 & $\mathbf{0 . 7 5}$ & 0.72 \\
5 & 0.80 & 0.58 & 0.63 & $\mathbf{0 . 6 9}$ \\
6 & 0.81 & $\mathbf{0 . 6 5}$ & 0.60 & 0.60 \\
7 & 0.82 & 0.61 & 0.68 & $\mathbf{0 . 7 2}$ \\
8 & 0.85 & 0.64 & 0.66 & $\mathbf{0 . 7 1}$ \\
9 & 0.87 & 0.67 & 0.71 & $\mathbf{0 . 7 3}$ \\
10 & 0.81 & 0.57 & 0.66 & $\mathbf{0 . 7 0}$ \\
11 & 0.70 & 0.60 & 0.65 & $\mathbf{0 . 6 7}$ \\
12 & 0.78 & 0.65 & $\mathbf{0 . 6 7}$ & 0.66 \\
13 & 0.88 & 0.60 & 0.64 & $\mathbf{0 . 6 6}$ \\
14 & 0.86 & 0.69 & $\mathbf{0 . 6 8}$ & $\mathbf{0 . 6 8}$ \\
15 & 0.90 & 0.67 & 0.67 & $\mathbf{0 . 7 0}$ \\
16 & 0.87 & 0.52 & $\mathbf{0 . 5 7}$ & 0.56 \\
\hline \hline mean & 0.82 & 0.62 & 0.65 & $\mathbf{0 . 6 7}$
\end{tabular}

Table 1: Classification accuracies across 16 subjects in a face vs. scrambled visual task. The column "single" reports the single-subject decoding accuracies (6 fold CV). The last three columns show leave-one-subject-out accuracies for pooling (pool), stacked generalization (SG) and stacked generalization with covariate shift $(\mathrm{SG}+\mathrm{CS})$. The last row shows the mean accuracies across subjects.

all subjects.

\section{Discussion and Conclusion}

In this paper, we formally presented the problem of MEG decoding across subjects for inferential purpose. We positioned the problem with respect to the current literature and in particular within the framework of transductive transfer learning (TTL). We presented a basic approach to TTL, i.e. simple covariate shift, based on trial weighting in order to penalize the training trials far from the test trials.

We draw an analogy between the problem of decoding across subjects and ensemble learning. We introduce stacked generalization as an ensembling method to deal the variability across the triaining subjects. The final method we proposed combines stacked generalization (SG) and covariate shift (CS).

The experiments presented in Section 3 compare the proposed approach to the baseline approach of simply pooling all the trials of all the training subjects without attempting to model their differences. The results in Table 1 show that both SG and CS are able to extract information from the similarities and differences between subjects. The baseline approach has average accuracy 0.62 
while SG alone reaches 0.65 and SG together with CS reach 0.67.

The results show a large decrease in accuracy between decoding across subjects and decoding single subjects, where accuracy reaches 0.82 on average. This gap motivates the need for further research.

\section{References}

[1] S. Lemm, B. Blankertz, T. Dickhaus, and K.-R. Müller, "Introduction to machine learning for brain imaging," NeuroImage, vol. 56, no. 2, pp. 387-399, May 2011. [Online]. Available: http://dx.doi.org/10.1016/j.neuroimage.2010.11.004

[2] E. Olivetti, S. Veeramachaneni, and E. Nowakowska, "Bayesian hypothesis testing for pattern discrimination in brain decoding," Pattern Recognition, May 2011. [Online]. Available: http://dx.doi.org/10.1016/j.patcog.2011.04.025

[3] J.-P. P. Kauppi, L. Parkkonen, R. Hari, and A. Hyvärinen, "Decoding magnetoencephalographic rhythmic activity using spectrospatial information." NeuroImage, vol. 83, pp. 921-936, Dec. 2013. [Online]. Available: http://dx.doi.org/10.1016/j.neuroimage.2013.07.026

[4] S. J. Pan and Q. Yang, "A Survey on Transfer Learning," Knowledge and Data Engineering, IEEE Transactions on, vol. 22, no. 10, pp. 1345-1359, Oct. 2010. [Online]. Available: http://dx.doi.org/10.1109/tkde.2009.191

[5] D. Devlaminck, B. Wyns, M. Grosse-Wentrup, G. Otte, and P. Santens, "Multisubject Learning for Common Spatial Patterns in Motor-Imagery BCI," Computational Intelligence and Neuroscience, vol. 2011, pp. 1-9, Jan. 2011. [Online]. Available: http://dx.doi.org/10.1155/2011/217987

[6] J. V. Haxby, J. S. Guntupalli, A. C. Connolly, Y. O. Halchenko, B. R. Conroy, M. I. Gobbini, M. Hanke, and P. J. Ramadge, "A common, high-dimensional model of the representational space in human ventral temporal cortex." Neuron, vol. 72, no. 2, pp. 404-416, Oct. 2011. [Online]. Available: http://dx.doi.org/10.1016/j.neuron.2011.08.026

[7] A. F. Marquand, M. Brammer, S. C. Williams, and O. M. Doyle, "Bayesian multi-task learning for decoding multi-subject neuroimaging data." NeuroImage, Feb. 2014. [Online]. Available: http://dx.doi.org/10.1016/j.neuroimage.2014.02.008

[8] H. Shimodaira, "Improving predictive inference under covariate shift by weighting the log-likelihood function," Journal of Statistical Planning and Inference, vol. 90, no. 2, pp. 227-244, Oct. 2000. [Online]. Available: http://dx.doi.org/10.1016/s0378-3758(00)00115-4 
[9] H. Daumé and D. Marcu, "Domain Adaptation for Statistical Classifiers," J. Artif. Int. Res., vol. 26, no. 1, pp. 101-126, May 2006. [Online]. Available: http://portal.acm.org/citation.cfm?id=1622562

[10] B. Zadrozny, "Learning and Evaluating Classifiers Under Sample Selection Bias," in Proceedings of the Twenty-first International Conference on Machine Learning, ser. ICML '04. New York, NY, USA: ACM, 2004, pp. 114+. [Online]. Available: http://dx.doi.org/10.1145/1015330.1015425

[11] L. I. Kuncheva, Combining Pattern Classifiers: Methods and Algorithms, 1st ed. Wiley-Interscience, Jul. 2004. [Online]. Available: http://www.worldcat.org/isbn/0471210781

[12] D. H. Wolpert, "Stacked generalization," Neural Networks, vol. 5, no. 2, pp. 241-259, Jan. 1992. [Online]. Available: http://dx.doi.org/10.1016/s0893-6080(05)80023-1

[13] K. M. Ting and I. H. Witten, "issues in Stacked Generalization," Journal of Artificial Intelligence Research, vol. 10, pp. 271-289, 1999. [Online]. Available: http://dx.doi.org/10.1613/jair.594

[14] R. N. Henson, D. G. Wakeman, V. Litvak, and K. J. Friston, "A Parametric Empirical Bayesian Framework for the EEG/MEG Inverse Problem: Generative Models for Multi-Subject and Multi-Modal Integration." Frontiers in human neuroscience, vol. 5, 2011. [Online]. Available: http://dx.doi.org/10.3389/fnhum.2011.00076 